\title{
BMJ Open Study protocol for a systematic review of evidence for digital interventions for comorbid excessive drinking and depression in community-dwelling populations
}

\author{
Bernd Schulte, ${ }^{1}$ Eileen F S Kaner, ${ }^{2}$ Fiona Beyer, ${ }^{2}$ Christiane S Schmidt, ${ }^{1}$ \\ Amy O'Donnell (D) ${ }^{2}$
}

To cite: Schulte B, Kaner EFS, Beyer F, et al. Study protocol for a systematic review of evidence for digital interventions for comorbid excessive drinking and depression in communitydwelling populations. BMJ Open 2019;9:e031503. doi:10.1136/ bmjopen-2019-031503

- Prepublication history and additional material for this paper are available online. To view please visit the journal (http:// dx.doi.org/10.1136/bmjopen2019-031503).

Received 08 May 2019 Revised 16 August 2019 Accepted 10 September 2019

Check for updates

(c) Author(s) (or their employer(s)) 2019. Re-use permitted under CC BY-NC. No commercial re-use. See rights and permissions. Published by BMJ.

${ }^{1}$ Centre of Interdisciplinary Addiction Research (ZIS), Department of Psychiatry and Psychotherapy, University Medical Center HamburgEppendorf, Hamburg, Germany ${ }^{2}$ Institute of Health and Society, Newcastle University, Newcastle upon Tyne, UK

Correspondence to

Dr Bernd Schulte;

B.Schulte@uke.de

\section{ABSTRACT}

Introduction Excessive drinking and depression are frequently comorbid and make a substantial contribution to the global non-communicable disease burden. $\mathrm{A}$ range of effective interventions and treatments exist for either excessive drinking or depression alone, including a positive emerging evidence base for the use of digital interventions. Computerised and/or smartphone delivered advice could provide flexible, coordinated support for patients with comorbid excessive drinking and depression. However, to date, no systematic review of the evidence has been conducted focused on the effectiveness of digital interventions for this specific comorbid population. This systematic review will identify and evaluate the effectiveness of digital interventions for reducing comorbid excessive drinking and depression in community-dwelling populations.

Methods and analysis We will search MEDLINE, The Cochrane Library, CENTRAL, CINAHL, PsycINFO, ERIC and SCl from inception to end of July 2019 for randomised controlled trials that evaluate any personalised digital intervention for comorbid excessive drinking and depression and published in any language. Primary outcomes will be changes in quantity of alcohol consumed and depressive symptoms. Screening, data extraction and risk of bias assessment will be undertaken independently by two reviewers, with disagreements resolved through discussion. Meta-analytic methods will be used to synthesise the data collected relating to the primary outcomes of interest.

Ethics and dissemination As a systematic review, ethical approval is not needed. Findings will be published in peerreviewed journals and presented at conferences. Trial registration number CRD42019130134.

\section{INTRODUCTION}

\section{Description of the condition}

Alcohol and mental health disorders make a substantial contribution to the global non-communicable disease burden. ${ }^{1}{ }^{2}$ Alcohol consumption alone is causally related to over 60 different medical conditions, ${ }^{3}$ with

\section{Strengths and limitations of this study}

- To our knowledge, this study represents the first systematic review focused specifically on the effectiveness of digital interventions for comorbid excessive drinking and depression.

- This review has the potential to inform the development of evidence-based interventions that could be delivered at scale to this at-risk population.

- The strengths of this systematic review include the use of an in-depth search strategy and robust quality appraisal criteria to identify and evaluate the existing literature.

- Potential limitations are likely to include between-study heterogeneity of the original studies and publication bias.

- Previous research in this field suggests that trials are likely to use a range of alcohol consumption and depression measures to assess outcomes.

excessive drinking associated with adverse social and economic consequences that extend beyond the individual drinker to their families, communities and society as a whole. ${ }^{4}$ Excessive drinking is defined here as either hazardous drinking, a pattern of alcohol consumption that increases an individual's risk of harmful consequences, ${ }^{5}$ or harmful drinking, a pattern that is causing mental or physical damage ${ }^{67}$ Excessive drinking is highly comorbid with a number of mental health conditions, including lifetime depression. ${ }^{8-10}$ In the UK, over two-fifths of people presenting with excessive drinking in primary care suffer from depression, ${ }^{11}$ and an estimated one in five hospital admissions for mental and behavioural disorders are due to alcohol. ${ }^{12}$ Experiencing such conditions comorbidly is associated with poorer overall outcomes for the individual concerned. Excessive drinking 
is connected with: worsening the depression course, with risks of incident depression higher for heavier as opposed to lighter drinkers, ${ }^{13}$ increased suicide risk, ${ }^{14}$ and delayed recovery from psychiatric conditions. ${ }^{15}$

A range of effective interventions and treatments exist for either excessive drinking or depression alone, including behavioural (typically psychotherapy) delivered either face-to-face or computerised support, and/ or pharmacological approaches. ${ }^{16}{ }^{17}$ In mild cases of depression, guided self-help and computerised cognitive behavioural therapy are recommended as initial treatments ${ }^{18}{ }^{19}$; while antidepressant drugs remain the mainstay of treatment for moderate to severe or sustained cases, particularly selective serotonin reuptake inhibitors. ${ }^{20}$ For excessive drinking, there is strong evidence for the effectiveness of brief behavioural advice for hazardous and harmful alcohol consumption delivered both face-to-face by primary care clinicians ${ }^{16}$ and digitally, via website or smartphone application. ${ }^{21}$ Specialist treatment is recommended for those drinking at dependent levels. ${ }^{22}$

For patients with comorbid excessive drinking and depression, the picture is more complex. Results for the use of cognitive-behavioural therapy and/or motivational interviewing for such individuals have been promising, demonstrating small but significant effects. ${ }^{23} 24$ However, there is limited conclusive evidence concerning whether parallel or integrated treatment models achieve better treatment outcomes for such patients, particularly over the longer term. ${ }^{25}$ Thus, it is likely that multifaceted, sustained interventions will be needed, delivered concomitantly, and closely monitored, to optimise their overall impact. ${ }^{26-28}$ Given the positive emerging evidence base for the use of digital interventions with excessive drinking and depression alone, ${ }^{21}$ 29-35 computerised and/or smartphone delivered advice and support could support the demand for flexible, more coordinated provision for patients experiencing such conditions comorbidly. Several trials suggest positive outcomes for digital interventions for comorbid excessive drinking and depression, ${ }^{36-40}$ but to date, no systematic review of the evidence has been conducted focused on this specific comorbid population. The proposed review aims to assess the effectiveness of digital interventions for reducing comorbid hazardous and harmful alcohol consumption and mild to moderate depression in community-dwelling populations.

\section{METHODS AND ANALYSIS}

This systematic review is registered with the International Prospective Register of Systematic Reviews (PROSPERO, https://www.crd.york.ac.uk/PROSPERO). The protocol has been written according to the Preferred Reporting Items for Systematic Review and Meta-Analysis Protocols recommendations ${ }^{41}$ and the findings will be reported using Preferred Reporting Items for Systematic Reviews and Meta-Analyses (PRISMA) guidelines. ${ }^{42}$

\section{Criteria for study inclusion}

Population

Studies must be performed among community-dwelling adults (18 years and older), who have been identified by themselves, significant others or via a validated screening process as having comorbid excessive drinking and depression, and have personally sought out or been directed towards any digital intervention for comorbid excessive drinking and depression. Excessive drinking is defined here as either hazardous or harmful drinking. ${ }^{5-7}$ Depression is defined as either major depression disorder, persistent depressive disorder or clinical depression assessed according to the WHO ICD-10 classification of mental and behavioural disorders ICD- $10^{7}$ or the Diagnostic and Statistical Manual of Mental Disorders (DSM$5)^{6}$ by a standardised interview (eg, Structured Clinical Interview, Composite International Diagnostic Interview) or via validated self-reports or rating scales with specific cut-off points for depression. Studies will be excluded if interventions are directed mainly towards people who are seeking specialist health or social care for alcohol dependence and/or severe depression (such as inpatient/residential programmes), or who were in treatment for, or recovery from, alcohol dependence (eg, 12-step programmes).

\section{Intervention}

Must be digital, defined as being delivered primarily through a programmable computer or mobile device (laptop, phone, or tablet), including web-based, mobile phone text messaging, smart-phone applications, social networking, or 'stand-alone' computer-based technologies (CD-ROMs) and must respond to user input and generate personalised content which aims to address the participants' alcohol-related behaviours and depression. Interventions which do not generate feedback or other output based on the personal characteristics of the user will not be included (eg, generic educational interventions). Interventions are not restricted to those accessible online.

\section{Comparator condition}

No intervention, usual care (in a health or social care setting), or other digital or face to face brief intervention to reduce alcohol consumption and depression.

\section{Outcomes}

The primary outcomes will be ${ }^{1}$ : quantity of alcohol consumed, which may be reported in standard drinks, alcohol units or similar, and which we will convert into grams of alcohol'; change in depressive symptoms, measured by a standardised or validated measure (Beck Depression Inventory (BDI ${ }^{43}$; Hamilton Depression Rating Scale (HAM-D), ${ }^{44}$ Patient Health Questionnaire, ${ }^{45}$ Depression Anxiety Stress Scales ${ }^{46}$ or any other depression scale). Where studies employ multiple validated rating scales for depression, preference would be for the BDI as a self-rating scale and for the HAM-D as 
an observer-rating scale. ${ }^{47}$ In acknowledgment of the varied outcome measures currently employed in these fields, ${ }^{48}$ we will also include the following secondary outcomes of relevance: number of drinking days; number of heavy drinking days; number of drinks per drinking day; number of days abstinent; total abstinence; time to relapse; quality of life (measured by the 36-item Short Form Health Survey ${ }^{50}$ or other validated tool); suiciderelated behaviour (measured by deaths by suicide, suicide attempts, episodes of deliberate self-harm ${ }^{51}$ ); and any reported adverse effects. To be eligible for inclusion, studies must report outcomes for both alcohol consumption and depression symptoms.

\section{Setting}

Participants may be recruited in a range of settings, including primary healthcare (including emergency departments), social care, educational, workplace or community. There is no restriction on where participants may interact with the intervention, given that it may be delivered through mobile devices.

\section{Study type}

Only randomised controlled trials (RCTs), with individual, cluster, stepped wedge and n-of one designs, will be eligible for inclusion. We will exclude cross-sectional studies, case series and case reports.

\section{Search strategy for identification of studies}

We will search the following electronic databases from inception to identify studies for inclusion in the review: MEDLINE (Ovid); The Cochrane Library (Wiley); CENTRAL (Cochrane Central Register of Controlled Trials); CINAHL (EBSCO); PsycINFO (Ovid); ERIC (EBSCO); and SCI (Science Citation Index via Web of Knowledge). Additionally, potentially eligible studies included in the recent Cochrane Review of Personalised digital interventions for reducing hazardous and harmful alcohol consumption ${ }^{21}$ will be identified from electronic databases held by the authors. We will check reference lists of all included studies and other relevant reviews, carry out citation searches for included studies, and consult experts to confirm nothing has been missed. The search will not be limited by publication status, language or date. An example of the proposed search strategy for MEDLINE is outlined in table 1.

\section{Study selection process}

Following de-duplication of the search results, two researchers will independently screen all titles and abstracts identified, using Endnote to ensure consistency in screening approach. The full research papers of any studies identified as being potentially eligible will be reviewed by two researchers independently. Any discrepancies will be resolved by discussion and by consulting a third researcher if necessary, to reach consensus. Reasons for exclusion from this phase of the search will be recorded. A PRISMA flow chart will outline the study selection process and reasons for exclusions.
Table 1 MEDLINE search strategy

\begin{tabular}{|c|c|}
\hline$\#$ & Searches \\
\hline 1 & exp Alcohol-Related Disorders/ \\
\hline 2 & exp Alcohol Drinking/ \\
\hline 3 & $\begin{array}{l}\text { (alcohol\$ adj2 (drink\$ or intoxicat } \$ \text { or use } \$ \text { or abus } \$ \text { or } \\
\text { misus } \$ \text { or risk } \$ \text { or consum } \$ \text { or withdraw } \$ \text { or detox } \$ \text { or } \\
\text { treat } \$ \text { or therap } \$ \text { or excess } \$ \text { or reduc } \$ \text { or cessation or } \\
\text { intervention } \$ \text { )).tw. }\end{array}$ \\
\hline 4 & $\begin{array}{l}\text { (drink\$ adj2 (excess or heavy or heavily or harm or harmful } \\
\text { or hazard } \$ \text { or binge or harmful or problem } \$ \text { )).tw. }\end{array}$ \\
\hline 5 & ("alcohol use" or alcoholic\$).tw. \\
\hline 6 & or/1-5 \\
\hline 7 & Depression/ \\
\hline 8 & exp Depressive Disorder/ \\
\hline 9 & Mood Disorders/ \\
\hline 10 & dysthymi\$.tw. \\
\hline 11 & (depressi\$ adj3 disorder\$).tw. \\
\hline 12 & (depressi\$ adj3 symptom\$).tw. \\
\hline 13 & mood disorder\$.tw. \\
\hline 14 & affective disorder\$.tw. \\
\hline 15 & antidepress\$.tw. \\
\hline 16 & anti-depress\$.tw. \\
\hline 17 & or/7-16 \\
\hline 18 & Internet/ \\
\hline 19 & Blogging/ \\
\hline 20 & Social Media/ \\
\hline 21 & Computers/ \\
\hline 22 & exp Microcomputers/ \\
\hline 23 & Minicomputers/ \\
\hline 24 & Therapy, Computer-Assisted/ \\
\hline 25 & Computer-Assisted Instruction/ \\
\hline 26 & exp Cellular Phone/ \\
\hline 27 & Electronic Mail/ \\
\hline 28 & $\begin{array}{l}\text { ((email\$ or e-mail\$ or electronic mail\$ or text messag } \$ \text { or } \\
\text { SMS or MMS or phone? or cellphone? or cell-phone? or } \\
\text { smartphone? or smart-phone? or digital tablet? or pda } \\
\text { or personal digital assistant? or social media or social } \\
\text { networking or facebook or twitter or skyp } \$ \text { or app?) adj3 } \\
\text { (deliver } \$ \text { or generat } \$ \text { or based or provid } \$ \text { or facilitat } \$ \text { or } \\
\text { support } \$ \text { or treatment? or therap } \$ \text { or intervention? or } \\
\text { program } \$ \text { or feedback)).ti,ab. }\end{array}$ \\
\hline 29 & $\begin{array}{l}\text { ((Internet\$ or electronic } \$ \text { or digital\$ or technolog } \$ \text { or online } \\
\text { or on-line or computer } \$ \text { or laptop? or software or web\$ } \\
\text { or weblog } \$ \text { or blog\$ or CD? or CD-ROM?) adj3 (deliver\$ } \\
\text { or generat } \$ \text { or based or provid } \$ \text { or facilitat } \$ \text { or support } \$ \\
\text { or treatment? or therap\$ or intervention? or program } \$ \text { or } \\
\text { feedback)).ti,ab. }\end{array}$ \\
\hline 30 & $\begin{array}{l}\text { (e-BI or e-SBI or ehealth or e-health or electronic health or } \\
\text { mhealth or m-health or mobile health or virtual health or } \\
\text { digital health or technological aid?).ti,ab. }\end{array}$ \\
\hline 31 & or/18-30 \\
\hline 32 & 6 and 17 and 31 \\
\hline 33 & randomized controlled trial.pt. \\
\hline 34 & controlled clinical trial.pt. \\
\hline 35 & randomi*.ab. \\
\hline
\end{tabular}

Continued 


\begin{tabular}{|c|c|}
\hline$\#$ & Searches \\
\hline 36 & placebo.ab. \\
\hline 37 & drug therapy.fs. \\
\hline 38 & randomly.ab. \\
\hline 39 & trial.ab. \\
\hline 40 & groups.ab. \\
\hline 41 & 33 or 34 or 35 or 36 or 37 or 38 or 39 or 40 \\
\hline 42 & (animals not (humans and animals)).sh. \\
\hline 43 & 41 not 42 \\
\hline 44 & 32 and 43 \\
\hline
\end{tabular}

\section{Data extraction}

Data will be extracted using a standardised data extraction form specifically developed and piloted for this study. Extracted data will include: study design and setting; sample size including recruitment and retention rates; participant characteristics; details of the intervention (including mode of delivery); primary and secondary outcome measures (including SD or related measures of variability) and information for the assessment of the risk of bias. Two researchers will carry out data extraction of each included study independently, with any discrepancies resolved by a third researcher. Where multiple eligible outcomes are recorded for depression, we will prioritise data from rating scales (eg, Hamilton Rating Scale for Depression) over self-report questionnaires (eg, BDI).

\section{Risk of bias}

Two researchers will independently assess the risk of bias of the included studies using the Cochrane Collaboration's tool for assessing risk of bias in RCTs ${ }^{52}$ For each included RCT, we will provide a description, comment and judgement of risk of bias for the following items ${ }^{1}$ : bias arising from the randomisation process ${ }^{2}$; bias due to deviations from intended interventions ${ }^{3}$; bias due to missing outcome data ${ }^{4}$; bias in measurement of the outcome ${ }^{5}$; bias in selection of the reported result (see online supplementary appendix 1 for details). These judgments will be informed by the criteria adapted to the addiction field by the Cochrane Collaboration. Blinding of participants, personnel and outcome assessor (avoidance of performance bias and detection bias) will be considered separately for objective outcomes (eg, drop out, use of substance of abuse measured by urine analysis, subjects relapsed at the end of follow-up, subjects engaged in further treatments) and subjective outcomes (eg, duration and severity of signs and symptoms of withdrawal, patient self-reported use of substance, side effects, social functioning as integration at school or at work, family relationship). Incomplete outcome data (avoidance of attrition bias) will be considered for all outcomes except for the drop out from the treatment, which is very often the primary outcome measure in trials on addiction.

\section{Synthesis of data and summary measures}

Data synthesis and meta-analysis

For all included RCTs, we will provide a detailed description of the results in both tables and text. If studies are sufficiently homogeneous to enable meta-analysis, we will pool the data for each outcome using a random-effects model in a meta-analysis that compares intervention and control arms, using mean differences or standardised mean differences as appropriate for continuous variables and relative risks for dichotomous outcomes. The meta-analysis will be performed using Review Manager (RevMan V5.3) developed by the Cochrane Community. If meta-analysis is not feasible, we will carry out a narrative summary of studies.

In the outcome assessment, for continuous variable outcomes (eg, quantity of alcohol consumed or scores in depression scales) we will compare standardised mean differences. For dichotomous outcomes (eg, participants classified as drinking over set limits or having depression remission), we will compare proportions using risk ratios. Where outcomes have been assessed at more than one time, data for each time point will be extracted. Depending on the availability of sufficient data, we will analyse follow-up durations using different time frames: (1) short term (up to 6 months postintervention); (2) medium term (6-12 months postintervention); and (3) long-term (more than 12 months postintervention).

\section{Unit of analysis issues}

For trials with more than one-and very similar-control or treatment arms, the results for these arms will be combined in the meta-analysis. If study arms cannot be combined, for example, due to important differences in intervention characteristics, each pair-wise comparison will be included separately. To avoid the multiple use of participants in the pooled estimate of treatment effect, every arm that is included more than once, will be divided by the number of comparisons where it is included.

For dichotomous outcomes, both the total number of patients and the number of events and will be divided up. For continuous outcomes, the means and SD will be left unchanged, and only the total number of participants will be divided. This method retains information from each arm of the trial while it compromises the precision of the pooled estimate slightly.

To allow the inclusion of cluster randomised trials with individually randomised trials in the same meta-analysis, we need to account for the relative variability within and between clusters. If a trial report only contains data that is not adjusted for the cluster design, we will add an external estimate of the intra-cluster coefficient to estimate a design effect, thus inflating the variance of the effect estimate.

\section{Dealing with missing data}

We will contact authors to try to obtain missing data. Where this is impossible, we will attempt to estimate primary outcome measures using secondary outcome 
measures; for example, estimating quantity of alcohol consumed using frequency and intensity of consumption. Trials with missing SD will be excluded from the main analysis for the associated continuous measure, but may be included in a sensitivity analysis, using imputed values for the SD.

\section{Assessment of heterogeneity}

Statistical heterogeneity will be assessed for significance with the Cochran's Q test statistic, and quantified with the $\mathrm{I}^{2}$ value. ${ }^{53}$ Causes of heterogeneity will be explored both narratively and using subgroup and sensitivity analyses.

Assessment of publication bias

We will evaluate publication bias using the Egger test and funnel plots. ${ }^{54}$

\section{Sensitivity analysis}

We will conduct sensitivity analyses by investigating the effect of restricting to studies with a low overall risk of bias.

\section{Patient and public involvement}

Patients and the public were not involved in the design and will be not involved in the conduct of the review. However, we will discuss the findings with the Newcastle Mental Health Service User Patient and Public Involvement Group and seek their assistance in interpreting the implications for policy and practice.

\section{Timeline}

For the complete systematic review and meta-analysis, a timeline of 9 months is foreseen (1 April 2019-31 December 2019).

\section{Ethics and dissemination}

As no primary data from studies will be collected, ethical approval is not needed for this systematic review. Findings will be published in peer-reviewed journals and presented at appropriate scientific conferences, congresses and symposia.

\section{CONCLUSION}

This review will examine the effectiveness of digital interventions for comorbid excessive drinking and depression. Digital interventions, delivered via computer or mobile phone, have the potential to provide cost-effective support for patients with excessive drinking and depression who may otherwise face socioeconomic and structural barriers to treatment. However, while previous studies have synthesised evidence of the impact of digital interventions on excessive drinking or depression alone, there is no comprehensive review that considers the effectiveness of such interventions in comorbid populations. This review will respond to this knowledge gap, and thus has the potential to inform the development of evidencebased interventions that could be delivered at scale to this at-risk population.
The strengths of this systematic review include the use of an in-depth search strategy and robust quality appraisal criteria to identify and evaluate the existing literature. However, potential limitations are likely to include between-study heterogeneity of the original studies and publication bias. Previous research in this field suggests that trials are likely to use a range of alcohol consumption and depression measures to assess outcomes ${ }^{55}$ Further, while we will endeavour to retrieve data from eligible unpublished and non-significant studies, our findings could be limited by publication bias. ${ }^{56}$

In conclusion, to our knowledge, this study represents the first systematic review of digital interventions for comorbid excessive drinking and depression. Findings will have relevance for healthcare practitioners and policy-makers, as well as helping to inform the direction of future research in this field.

Contributors $B S$ and $A 0^{\prime} D$ designed the initial review concept and drafted the manuscript. EFSK, FB and CSS provided critical insights to the protocol including search methods, data extraction processes and methodological appraisal of the studies. All authors contributed to the revised manuscript and provided their consent.

Funding The authors have not declared a specific grant for this research from any funding agency in the public, commercial or not-for-profit sectors.

Competing interests None declared.

Patient consent for publication Not required.

Provenance and peer review Not commissioned; externally peer reviewed.

Open access This is an open access article distributed in accordance with the Creative Commons Attribution Non Commercial (CC BY-NC 4.0) license, which permits others to distribute, remix, adapt, build upon this work non-commercially, and license their derivative works on different terms, provided the original work is properly cited, appropriate credit is given, any changes made indicated, and the use is non-commercial. See: http://creativecommons.org/licenses/by-nc/4.0/.

ORCID iD

Amy 0'Donnell http://orcid.org/0000-0003-4071-9434

\section{REFERENCES}

1 Lim SS, Vos T, Flaxman AD, et al. A comparative risk assessment of burden of disease and injury attributable to 67 risk factors and risk factor clusters in 21 regions, 1990-2010: a systematic analysis for the global burden of disease study 2010. The Lancet 2012;380:2224-60.

2 Whiteford HA, Ferrari AJ, Degenhardt L, et al. The global burden of mental, neurological and substance use disorders: an analysis from the global burden of disease study 2010. PLoS One 2015;10:e0116820.

3 Rehm J, Mathers C, Popova S, et al. Global burden of disease and injury and economic cost attributable to alcohol use and alcohol-use disorders. The Lancet 2009;373:2223-33.

4 World Health Organisation. Global status report on alcohol and health. Geneva: World Health Organisation, 2011.

5 NICE. Alcohol-use disorders: preventing the development of hazardous and harmful drinking: NICE public health guidance 24 . London: National Institute for Health and Clinical Excellence, 2010.

6 American Psychiatric Association. Diagnostic and statistical manual of mental disorders (DSM-5®): American psychiatric PUB 2013.

7 World Health Organisation. International statistical classification of diseases and related health problems. 10th edn. Geneva: World Health Organisation, 2007.

8 Odlaug BL, Gual A, DeCourcy J, et al. Alcohol dependence, cooccurring conditions and attributable burden. Alcohol and Alcoholism 2016;51:201-9.

9 HMX L, Cleary M, Sitharthan T, et al. Prevalence of comorbid substance use, anxiety and mood disorders in epidemiological 
surveys, 1990-2014: a systematic review and meta-analysis. Drug Alcohol Depend 2015;154:1-13.

10 Grant BF, Goldstein RB, Saha TD, et al. Epidemiology of DSM5 alcohol use disorder: results from the National epidemiologic survey on alcohol and related conditions III. JAMA Psychiatry 2015;72:757-66.

11 PHE. Better care for people with co-occurring mental health and alcohol/drug use conditions. London: Public Health England, 2017.

12 PHE. The public health burden of alcohol and the effectiveness and cost-effectiveness of alcohol control policies: an evidence review. public health England, 2016. Available: https://www.gov.uk/ government/uploads/system/uploads/attachment_data/file/583047/ alcohol_public_health_burden_evidence_review.pdf

13 Bellos S, Skapinakis P, Rai D, et al. Longitudinal association between different levels of alcohol consumption and a new onset of depression and generalized anxiety disorder: results from an international study in primary care. Psychiatry Res 2016;243:30-4.

14 Rehm J, Gmel GE, Gmel G, et al. The relationship between different dimensions of alcohol use and the burden of disease-an update. Addiction 2017;112:968-1001.

15 Greenfield TK. Chapter 21: Individual Risk of Alcohol-Related Disease and Problems. In: Heather N, Peter TJ, Stockwell T, eds. International Handbook alcohol dependence and problems. John Wiley \& Sons Ltd, 2001: 413-39.

16 Kaner EFS, Beyer FR, Muirhead C, et al. Effectiveness of brief alcohol interventions in primary care populations. Cochrane Database Syst Rev 2018;24.

17 Linde K, Sigterman K, Kriston L, et al. Effectiveness of psychological treatments for depressive disorders in primary care: systematic review and meta-analysis. The Annals of Family Medicine 2015;13:56-68

18 Cuijpers P, Donker T, van Straten A, et al. Is guided self-help as effective as face-to-face psychotherapy for depression and anxiety disorders? A systematic review and meta-analysis of comparative outcome studies. Psychol Med 2010;40:1943-57.

19 National Collaborating Centre for Mental Health. Depression: the NICE guideline on the treatment and management of depression in adults (updated version). National clinical practice guideline 90. London: National Collaborating Centre for Mental Health commisioned by NICE, 2018.

20 NICE. Depression: management of depression in primary and secondary care - NICE quidance. London: National Institute for Clinical Excellence, 2010.

21 Kaner EFS, Beyer FR, Garnett C, et al. Personalised digital interventions for reducing hazardous and harmful alcohol consumption in community-dwelling populations. Cochrane Database Syst Rev 2017;48.

22 NICE. Alcohol-use disorders: diagnosis, assessment and management of harmful drinking and alcohol dependence (CG115. London: National Institute for Health and Care Excellence, 2011.

23 Riper H, Andersson G, Hunter SB, et al. Treatment of comorbid alcohol use disorders and depression with cognitive-behavioural therapy and motivational interviewing: a meta-analysis. Addiction 2014;109:394-406.

24 Nunes EV, Levin FR. Treatment of depression in patients with alcohol or other drug dependence. JAMA 2004;291:1887-96.

25 Hobden B, Bryant J, Carey M, et al. Finding the optimal treatment model: a systematic review of treatment for co-occurring alcohol misuse and depression. Aust N Z J Psychiatry 2018;52:737-50.

26 Kelly TM, Daley DC, Douaihy AB. Treatment of substance abusing patients with comorbid psychiatric disorders. Addict Behav 2012;37:11-24.

27 Baker AL, Kavanagh DJ, Kay-Lambkin FJ, et al. Randomized controlled trial of MICBT for co-existing alcohol misuse and depression: outcomes to 36-months. J Subst Abuse Treat 2014;46:281-90.

28 Baker AL, Thornton LK, Hiles S, et al. Psychological interventions for alcohol misuse among people with co-occurring depression or anxiety disorders: a systematic review. J Affect Disord 2012;139:217-29.

29 Donker T, Petrie K, Proudfoot J, et al. Smartphones for smarter delivery of mental health programs: a systematic review. J Med Internet Res 2013;15:e247-e.

30 Andersson G, Cuijpers P, Carlbring P, et al. Guided Internet-based vs face-to-face cognitive behavior therapy for psychiatric and somatic disorders: a systematic review and meta-analysis. World Psychiatry 2014:13:288-95.

31 Davies EB, Morriss R, Glazebrook C. Computer-delivered and web-based interventions to improve depression, anxiety, and psychological well-being of university students: a systematic review and meta-analysis. J Med Internet Res 2014;16:e130.
32 Griffiths KM, Farrer L, Christensen H. The efficacy of internet interventions for depression and anxiety disorders: a review of randomised controlled trials. Medical Journal of Australia 2010;192:S4-11.

33 Riper $\mathrm{H}$, Hoogendoorn A, Cuijpers P, et al. Effectiveness and treatment moderators of internet interventions for adult problem drinking: an individual patient data meta-analysis of 19 randomised controlled trials. PLoS Med 2018;15:e1002714.

34 K. Nair N, C. Newton N, Shakeshaft A, et al. A systematic review of digital and computer-based alcohol intervention programs in primary care. Current drug abuse reviews 2015;8:111-8.

35 Khadjesari Z, Murray E, Hewitt C, et al. Can stand-alone computerbased interventions reduce alcohol consumption? A systematic review. Addiction 2011;106:267-82.

36 Deady M, Mills KL, Teesson M, et al. An online intervention for cooccurring depression and problematic alcohol use in young people: primary outcomes from a randomized controlled trial. $J$ Med Internet Res 2016;18:e71.

37 Deady M, Teesson M, Kay-Lambkin F, et al. Evaluating a brief, Internet-based intervention for co-occurring depression and problematic alcohol use in young people: protocol for a randomized controlled trial. JMIR Res Protoc 2014;3:e6-e.

38 Kay-Lambkin FJ, Baker AL, Lewin TJ, et al. Computer-based psychological treatment for comorbid depression and problematic alcohol and/or cannabis use: a randomized controlled trial of clinical efficacy. Addiction 2009;104:378-88.

39 Geisner IM, Varvil-Weld L, Mittmann AJ, et al. Brief web-based intervention for college students with comorbid risky alcohol use and depressed mood: does it work and for whom? Addict Behav 2015;42:36-43.

40 Deady M, Kay-Lambkin F, Teesson M, et al. Developing an integrated, Internet-based self-help programme for young people with depression and alcohol use problems. Internet Interventions 2014:1:118-31.

41 Shamseer L, Moher D, Clarke M, et al. Preferred reporting items for systematic review and meta-analysis protocols (PRISMA-P) 2015 elaboration and explanation. BMJ 2015;349:97647.

42 Liberati A, Altman DG, Tetzlaff J, et al. The PRISMA statement for reporting systematic reviews and meta-analyses of studies that evaluate health care interventions: explanation and elaboration. PLoS Med 2009;6:e1000100

43 Beck AT, Steer RA, Brown GK. Beck depression inventory-II. San Antonio, 1996: 78. 490-82.

44 Hamilton M. The Hamilton rating scale for depression. assessment of depression. Springer, 1986: 143-52.

45 Kroenke K, Spitzer RL, Williams JB. The PHQ-9: validity of a brie depression severity measure. J Gen Intern Med 2001;16:606-13.

46 Lovibond PF, Lovibond SH. The structure of negative emotional states: comparison of the depression anxiety stress scales (DASS) with the Beck depression and anxiety inventories. Behav Res Ther 1995;33:335-43.

47 Shinohara K, Honyashiki M, Imai H, et al. Behavioural therapies versus other psychological therapies for depression. Cochrane Database Syst Rev 2013;138.

48 Shorter GW, Bray JW, Giles EL, et al. The variability of outcomes used in efficacy and effectiveness trials of alcohol brief interventions: a systematic review. J Stud Alcohol Drugs 2019;80:286-98.

49 Shorter GW, Heather N, Bray JW, et al. Prioritization of outcomes in efficacy and effectiveness of alcohol brief intervention trials: international Multi-Stakeholder e-Delphi consensus study to inform a core outcome set. J Stud Alcohol Drugs 2019;80:299-309.

50 Ware JE, Sherbourne CD. The mos 36-item short-form health survey (SF-36): I. conceptual framework and item selection. Medical care 1992;30:473-83

51 Osman A, Bagge CL, Gutierrez PM, et al. The Suicidal Behaviors Questionnaire-Revised (SBQ-R):Validation with Clinical and Nonclinical Samples. Assessment 2001;8:443-54.

52 Higgins J, Sterne J, Savović J, et al. Editors. A revised tool for assessing risk of bias in randomized trials. Cochrane Database of Systematic Reviews 2016.

53 Higgins JPT, Thompson SG, Deeks JJ. Measuring inconsistency in meta-analyses. BMJ 2003;327:557-60.

54 Egger M, Smith GD, Schneider M, et al. Bias in meta-analysis detected by a simple, graphical test. BMJ 1997;315:629-34.

55 Shorter GW, Heather N, Bray JW, et al. The 'Outcome Reporting in Brief Intervention Trials: Alcohol' (ORBITAL) framework: protocol to determine a core outcome set for efficacy and effectiveness trials of alcohol screening and brief intervention. Trials 2017;18:611.

56 loannidis JPA. Why most published research findings are false. PLoS Med 2005;2:e124. 